Assurances et gestion des risques

Insurance and Risk Management

\title{
Repenser « l'esprit du capitalisme » : une rétrospective
}

\section{Nabil Khoury}

Volume 86, numéro 2-3, décembre 2019

URI : https://id.erudit.org/iderudit/1068505ar

DOI : https://doi.org/10.7202/1068505ar

Aller au sommaire du numéro

\section{Éditeur(s)}

Faculté des sciences de l'administration, Université Laval

ISSN

1705-7299 (imprimé)

2371-4913 (numérique)

Découvrir la revue

Citer cet article

Khoury, N. (2019). Repenser « l'esprit du capitalisme » : une rétrospective. Assurances et gestion des risques / Insurance and Risk Management, 86(2-3), 165-190. https://doi.org/10.7202/1068505ar
Résumé de l'article

Max Weber introduit l'idée que le système capitaliste de production s'est développé dans les communautés calvinistes du XVII ${ }^{\mathrm{e}}$ siècle les rendant plus prospères que les communautés catholiques contemporaines, et ce grâce à l'apport indispensable d'un " esprit de capitalisme » fondé sur une éthique calviniste austère étrangère au catholicisme. Cette théorie demeure un point de référence incontournable auprès des chercheurs depuis bientôt cent ans. L'objet de cet article est de présenter une synthèse des contributions théoriques et empiriques qui ont marqué, surtout au cours des cinq dernières décennies, l'évolution de la recherche sur ce thème. Nous situerons ces contributions dans le courant progressiste de l'évolution de la pensée économique sur ce sujet, afin de mieux apprécier les nouvelles orientations de la recherche dans ce domaine.
Tous droits réservés $\odot$ Faculté des sciences de l'administration, Université Laval, 2020
Ce document est protégé par la loi sur le droit d'auteur. L’utilisation des services d'Érudit (y compris la reproduction) est assujettie à sa politique d'utilisation que vous pouvez consulter en ligne.

https://apropos.erudit.org/fr/usagers/politique-dutilisation/ 


\section{REPENSER «L'ESPRIT DU CAPITALISME»: UNE RÉTROSPECTIVE}

Nabil KHOURY ${ }^{1}$

\section{RÉSUMÉ}

Max Weber introduit l'idée que le système capitaliste de production s'est développé dans les communautés calvinistes du XVII siècle les rendant plus prospères que les communautés catholiques contemporaines, et ce grâce à l'apport indispensable d'un "esprit de capitalisme» fondé sur une éthique calviniste austère étrangère au catholicisme. Cette théorie demeure un point de référence incontournable auprès des chercheurs depuis bientôt cent ans. L'objet de cet article est de présenter une synthèse des contributions théoriques et empiriques qui ont marqué, surtout au cours des cinq dernières décennies, l'évolution de la recherche sur ce thème. Nous situerons ces contributions dans le courant progressiste de l'évolution de la pensée économique sur ce sujet, afin de mieux apprécier les nouvelles orientations de la recherche dans ce domaine.

\section{ABSTRACT}

Max Weber argued that the capitalist system of production emerged in the Calvinist communities of the XVII century and made them more prosperous than the contemporaneous catholic communities, because they developed a "spirit of capitalism" based on a Calvinist ethic unfamiliar to Catholicism. His theory has opened new perspectives and has stimulated research for the last hundred years. This paper presents a contextualized review of the theoretical and empirical contributions that marked the advancement of research on this topic, more particularly during the last five decades. We position these contributions in the broader context of the evolution of economic thought in this field in order to appreciate the new orientation of research in this regard. 


\section{INTRODUCTION}

L'analyse de la relation entre l'Ethos du protestantisme ou plus précisément du calvinisme du $\mathrm{XVI}^{\mathrm{e}}$ siècle d'une part et de la croissance économique d'autre part, continue à alimenter le débat entamé il y a plus de cent ans autour de cette question. Un grand nombre de penseurs anciens et contemporains ont offert des réflexions incisives sur ce phénomène en vue de l'analyser dans sa complexité à travers le prisme subjectif des forces qui ont conduit à l'émergence du capitalisme moderne et corollairement la croissance économique dans les communautés protestantes et non dans les communautés catholiques contemporaines. Parmi les nombreuses explications avancées, celle du sociologue, économiste et politicien allemand Max Weber développée dans son livre "L'éthique protestante et l'esprit du capitalisme» est considérée comme fondamentale puisqu'elle a établi le cadre de référence pour toutes les analyses subséquentes ${ }^{2}$. Reprise par les uns pour démontrer sa validité, modifiée à divers degrés et même rejetée catégoriquement par les autres, sa thèse originale, à l'effet que le système de production capitaliste moderne n'aurait pas pu se développer au XVII ${ }^{e}$ siècle sans l'apport d'un "esprit capitaliste» fondé sur une éthique calviniste austère pour le soutenir, demeure un point de référence incontournable auprès des chercheurs ${ }^{3}$. Cette thèse se prête à plusieurs interprétations qui vont même jusqu'à l'utiliser à l'encontre de la théorie du "matérialisme historique" de Karl Marx à l'effet que toutes les institutions humaines, incluant la religion, ont un fondement économique, alors que la théorie de Weber réintroduit la question religieuse autrement que comme tributaire de l'infrastructure économique et laisse entrevoir que c'est plutôt un mouvement religieux qui pourrait être à la base du capitalisme moderne et non l'inverse ${ }^{4}$.

D'entrée de jeu, il convient de clarifier si la relation originale entre l'éthique calviniste et "l'esprit du capitalisme", développée par Max Weber dans sa thèse, est causale ou plutôt conjoncturelle comme le souligne d'ailleurs Marcel Hénaff'. À cet égard, la presque totalité des chercheurs qui se sont penchés sur cette théorie ont interprété ce lien comme étant causal et direct. Il convient cependant de porter une attention à la complexité de cette relation, comme Weber l'a d'ailleurs soulignée dans sa thèse. En effet, Weber observe que, d'un point de vue historique, les régions les plus développées économiquement en Allemagne, donc celles qui ont adopté «l'esprit du capitalisme» qu'il décrit, sont celles qui étaient à l'avant-garde de la Réforme de l'Église, ce qui peut porter à croire que le développement de l'esprit capitaliste fut le mobile de la Réforme religieuse et non l'inverse. Cependant, un 
tel lien causal ne peut résister à l'analyse puisque la Réforme n'a pas réduit mais a plutôt augmenté l'emprise de l'Église sur les fidèles, ce qui est paradoxal car le progrès économique tend généralement à libérer les individus de la domination du clergé. Ceci porte Weber à préconiser dans certaines conditions une conjonction de fait entre l'éthique calviniste et "l'esprit du capitalisme». Malgré la complexité apparente de la relation, toutes les études révisionnistes et empiriques qui ont porté sur le lien original développé par Weber entre l'éthique calviniste et "l'esprit du capitalisme", et qui sont répertoriées dans cette étude, ont interprété ce lien comme étant causal et direct, et c'est cette interprétation que nous adoptons dans ce travail.

L'objet de cet article est donc de faire une synthèse des contributions théoriques et empiriques qui ont marqué, surtout au cours des six dernières décennies, l'évolution de la recherche sur la théorie de Weber. Conscient du risque d'une telle limitation de la riche tradition scientifique relative à ce thème, nous avons situé ces contributions importantes dans le courant progressiste de l'évolution de la pensée économique sur ce sujet, afin de mieux apprécier les nouvelles orientations de la recherche dans ce domaine. À cette fin, la deuxième partie de l'article se concentre sur la théorie de "l'esprit du capitalisme» de Weber en vue de dégager le cadre conceptuel de l'analyse conduisant à la relation entre la religion et le progrès économique. La troisième partie est centrée sur les orientations révisionnistes et empiriques des études qui ont marqué les étapes importantes de l'évolution du débat autour de cette théorie. Comme l'analyse le montrera, la question est fort complexe et a poussé les chercheurs à poser des questions de plus en plus profondes pour éclairer le sujet. La quatrième et dernière partie présente nos conclusions sur l'état d'avancement de la recherche dans ce domaine et sur son évolution future.

\section{2. «L'ESPRIT DU CAPITALISME »}

L'Ethos du calvinisme austère du XVII ${ }^{\mathrm{e}}$ siècle a-t-il été un élément déclencheur d'un "esprit capitaliste" qui a favorisé l'émergence d'un système de production capitaliste et de son corollaire, la croissance économique, dans les communautés protestantes de l'Europe du Nord, alors que le catholicisme a freiné l'implantation de ce processus capitaliste dans les communautés catholiques contemporaines du sud de l'Europe? La question est importante car elle permet d'élucider le lien entre la religion et le développement économique et social des peuples. 
Weber analyse ce sujet à une époque où la religion dominait la vie de tous les jours. Il observe alors qu'avant l'arrivée du protestantisme, la poursuite de la richesse était considérée contraire à l'éthique religieuse du temps. L'activité économique, qui était reléguée au second plan après la vocation religieuse, était alors dominée par des métiers traditionnels qui ne permettaient ni un enrichissement financier individuel ni le salut de l'âme. À l'encontre de ces croyances, Martin Luther (14831546) avança l'idée que le salut ne s'obtient que par la grâce de Dieu et que le fait de se consacrer à un travail quel qu'il soit avec persévérance et diligence est une manifestation de cette grâce. Ainsi, toutes les vocations qu'elles soient religieuses ou économiques s'équivalent. Jean Calvin (1509-1564) poussa cette doctrine plus loin encore en spécifiant que la grâce de Dieu était un don gratuit qui est accordé aux personnes prédestinées selon la volonté divine qui est insondable. Et comme on ne peut savoir qui est prédestiné, l'idée s'est développée dans la communauté calviniste que le mode de vie est indicatif de la prédestination $^{6}$. Plus précisément, la réalisation totale de soi dans le travail et la frugalité sont deux importantes qualités que l'individu doit développer car ils reflètent une prédestination à être sauvé par la grâce de Dieu. Ainsi, les bonnes ouvres, le jeûne, les sacrements, les mortifications, la soumission aux autorités cléricales etc. ne peuvent permettre d'obtenir la grâce de Dieu, car ils ne peuvent servir de monnaie d'échange contre un don gratuit. Par contre, l'accumulation de richesse grâce à une carrière réussie, donne confiance en la grâce salvatrice de Dieu sans avoir à recourir à l'approbation du clergé. Cette nouvelle éthique protestante remettait ainsi en question les notions catholiques de vocation religieuse et de salut.

L'éthique protestante dont il est question ici mérite d'être approfondie. D'entrée de jeu, il importe de préciser que cette éthique ne représente pas un dogme ou une croyance religieuse, mais plutôt une attitude religieuse qui a pris beaucoup d'importance chez les calvinistes. L'élément fondamental de cette éthique est que tout travail qu'il soit à un petit salaire ou qu'il soit source de richesse pour l'entrepreneur, est perçu comme une vocation (Beruf) ou appel de Dieu tel que défini par Luther, et constitue par le fait même l'unique chemin qui mène au royaume de Dieu, c'est-à-dire au salut. Selon certains auteurs dont Charles Taylor ${ }^{7}$, cette affirmation des vertus de la vie ordinaire ne s'est pas manifestée soudainement, mais semble plutôt avoir suivi un processus progressif assez lent dans le puritanisme. L'idée nouvelle dans cette notion d'éthique est que l'accomplissement des devoirs correspondant au rôle que la providence a assigné à l'individu dans la vie, constitue la seule façon de vivre qui est agréable à Dieu et qui peut 
attester que l'individu est parmi ceux qui sont sauvés. Ainsi, le travail quotidien qui est destiné à chaque individu prend une signification religieuse et devient une vocation (Beruf) pour sauver l'âme. Cette double notion religieuse de destinée et de vocation au travail de chacun, conduit à ce que la poursuite de la richesse devient un objectif louable, atteignable à travers un effort inlassable et un dévouement total au travail ainsi qu'à travers un resserrement des dépenses de consommation, des dons de charité, et l'adhésion au principe d'honnêteté dans les relations avec autrui. Par contre, les distractions sociales ainsi que le confort et les plaisirs des biens matériels sont généralement proscrits.

Cette idée de l'éthique a été adoptée par les calvinistes qui en ont fait une caractéristique importante de leur philosophie ${ }^{8}$. Pour eux, le monde existe uniquement pour réaliser la gloire de Dieu. Ainsi, la division du travail présentée par Luther comme une expression de l'amour du prochain, puisque la division du travail oblige chaque individu à travailler pour les autres, devient pour les calvinistes une caractéristique importante de leur système d'éthique. Le travail professionnel doit ainsi servir la vie de la communauté puisque Dieu veut que la vie sociale soit organisée selon Ses commandements et pour Sa gloire. Autrement dit, mettre le travail au service de la communauté rend gloire à Dieu. Dans ce sens, même l'occupation la plus piètre est honorée par Dieu car elle contribue au bien-être collectif.

Ces éléments théologiques spécifiques du calvinisme, particulièrement "la doctrine de la destinée ", c'est-à-dire du rôle assigné à chaque personne par Dieu, et celle de "la vocation séculaire", c'est-à-dire que le travail quotidien quel qu'il soit s'élève au niveau d'une vocation religieuse (Beruf), font de cette branche du protestantisme une force génératrice de l'esprit capitaliste sous-jacent au système de production capitaliste moderne ${ }^{9}$. Ainsi, chaque métier et chaque profession représentent pour son titulaire une fin en soi pour laquelle il doit être dévoué. Dans ce même esprit, le calvinisme ainsi que les autres tendances protestantes austères interdisent le gaspillage de la richesse de même que l'engouement pour le luxe. Ils interdisent aussi les dons généreux aux églises et aux congrégations et considèrent que l'aumône aux pauvres encourage la paresse de ces derniers, ce qui est un péché puisque le travail est glorifié par Dieu ${ }^{10}$. Cette culture religieuse, une fois disséminée dans la population, prend racine et est transmise, selon Weber, de génération en génération même après que le fondement religieux sous-jacent disparait. 
Max Weber se penche ensuite sur le système capitaliste ${ }^{11}$ industriel moderne qui se caractérise par une organisation méthodique et rationnelle du travail afin de générer des profits. Ce système qui a percé dans les milieux qui pratiquaient le protestantisme austère ne pouvait exister, selon Weber, sans l'émergence d'un "esprit capitaliste" qui favorise la poursuite du profit pour réaliser le salut de l'âme. Cet esprit capitaliste semble si anormal que, pour se répandre, il devait s'appuyer sur une éthique protestante, plus particulièrement sur l'éthique calviniste qui favorise l'ascétisme, tel que défini précédemment en lien avec le travail comme vocation religieuse. Sous l'influence de cette éthique du $\mathrm{XVII}^{\mathrm{e}}$ siècle, les populations de l'Europe protestante se sont engagées dans le développement d'entreprises, dans le commerce et dans l'accumulation de richesses pour l'investissement. Dans ce sens, l'éthique protestante a alimenté l'attitude et les objectifs, autrement dit l'esprit capitaliste des populations concernées, ce qui a constitué une force importante dans l'émergence de ce capitalisme moderne.

Étant donné que la science et la technologie jouent un rôle important dans la culture capitaliste, on peut présumer, à l'instar de Weber, qu'il existe une corrélation positive entre les avancées scientifiques et le calvinisme au XVII ${ }^{\mathrm{e}}$ siècle. Robert $\mathrm{K}$. Merton a adressé cette question et conclut dans son étude que dans le Calvinisme du XVII ${ }^{\mathrm{e}}$ siècle, le rationalisme n'était pas incompatible avec la foi. En effet, en prônant l'esprit rationaliste et l'apprentissage des sciences expérimentales, l'éthos calviniste a sanctionné l'étude des phénomènes naturels comme un moyen efficace pour promouvoir la gloire de Dieu. En ce sens, la religion devenait un facteur important de l'avancement des connaissances scientifiques: les réalisations scientifiques étaient désormais perçues, selon Merton, comme une manifestation de la gloire de Dieu pour le bien de l'humanité ${ }^{12}$, ce qui valide l'hypothèse de Weber.

Le développement d'un tel esprit capitaliste ne s'est pas produit, selon Weber, dans les pays catholiques même si la pensée capitaliste a connu un essor non-négligeable au fil des siècles dans ces communautés. À titre d'exemple, les scholastiques, et surtout Saint Thomas D'Aquin, ont formulé une des premières théories économiques en Occident pour justifier, entre autres, la rémunération des prêts d'affaires en contrepartie du risque d'investissement auquel le créancier s'expose. Aussi, Alberti de Florence, avait déjà défini, au XVe siècle, les vertus bourgeoises qu'on retrouve plus tard chez les calvinistes puritains ${ }^{13}$. De plus, la célèbre admonition de Saint Paul: "Si quelqu'un ne veut pas travailler, qu'il ne mange pas non plus» était bien connue chez les catholiques comme chez les protestants et a été interprétée de plusieurs 
façons pour accommoder diverses situations, ce qui lui a fait perdre son mordant. Mais ces exemples anecdotiques et bien d'autres n'ont pas modifié, selon Weber, l'éthique de fraternité ancrée dans les communautés latines catholiques laquelle ne favorise pas le développement d'un esprit capitaliste, tel que défini précédemment.

Par ailleurs, Weber observe que même dans les pays où cet esprit capitaliste était important, d'autres formes de capitalisme non fondées sur l'éthique du protestantisme austère pouvaient émerger. À titre d'exemple, le système de capitalisme spéculatif, ou celui du capitalisme aventurier ou même du capitalisme colonial pouvaient exister et être dirigés par des magnats qui n'adoptaient pas le style austère du calvinisme. Cependant, la théorie de Weber ne s'applique qu'au système capitaliste de production caractérisé par une organisation méthodique et rationnelle du travail en vue de générer de la richesse et qui est encadré par un esprit capitaliste fondé sur le calvinisme austère.

Il convient de souligner que la théorie de Weber est basée sur un argument conjectural qui s'appuie sur une perception du comportement que les individus auraient dû adopter, selon l'auteur, s'ils avaient respecté les principes de leurs croyances religieuses. Il s'agit d'un argument subjectif difficile à commenter puisqu'il reflète pour l'auteur l'attitude plausible des calvinistes ${ }^{14}$. De plus, selon Viner, la plupart des théologiens calvinistes traditionnalistes considèrent que la définition de l'éthique calviniste de Weber ne concorde pas avec les enseignements de Calvin ni avec la vie des premiers calvinistes. Tout au plus, certains théologiens l'ont acceptée comme reflétant les formes dégénérées du calvinisme qui se sont manifestées plus tard dans l'histoire. De façon générale, la théorie de Weber a été reçue dans plusieurs milieux académiques comme une explication plausible, simple et cohérente de l'émergence de l'esprit capitaliste chez les protestants pratiquant le calvinisme austère au XVII ${ }^{\mathrm{e}}$ siècle $^{15}$.

Un aspect intéressant de la théorie de Weber mérite toutefois d'être souligné. Il s'agit de la résistance des communautés catholiques au processus du développement capitaliste. Weber invoque cette situation sans l'analyser. Dans une étude, Marcel Hénaff a procédé à l'élargissement du cadre analytique posé par Weber en y incluant une explication de la non-émergence du système capitaliste chez les catholiques. Pour ce faire, Hénaff prône une approche analytique positive plutôt que des observations circonstancielles. Aussi, après un examen approfondi sur le plan théologique de la thèse de Weber et de la tradition religieuse catholique de l'époque, Hénaff conclut que l'attitude négative des 
catholiques vis-à-vis le capitalisme émanait plutôt de leur culture sociale qui préconisait le principe qu'on pouvait retourner la grâce de Dieu en faisant la charité. Il argumente, à la suite de Clavero, que cette culture donnait préséance à une organisation sociale dont les relations sont fondées sur le soutien mutuel et la charité plutôt que sur les rapports contractuels légaux. Dans un tel contexte, la filiation et le copinage l'emportent sur le respect de la loi et de l'ordre, et la notion d'égalité proportionnelle remplace celle de l'égalité commutative. Cette culture des communautés catholiques était à l'opposé de celle des communautés protestantes et s'est avérée incompatible avec les besoins du capitalisme dans sa phase émergente.

\section{INVESTIGATION SCIENTIFIQUE}

Au fil des cent dernières années, plusieurs chercheurs, issus de disciplines différentes, se sont penchés sur la théorie de Weber. La controverse qui s'en est suivie a poussé l'investigation scientifique vers des questions de plus en plus profondes entourant cette théorie. La plupart des chercheurs ont examiné la théorie soit sous l'angle d'une analyse révisionniste ou sous celui d'une analyse empirique. Dans les paragraphes qui suivent, nous faisons un tour d'horizon des récentes études qui ont marqué les principaux courants de la controverse, tout en faisant une distinction entre les deux approches analytiques précitées. Notre objectif sera de renforcer la compréhension des nouvelles orientations de la recherche sur le rôle de la religion dans la croissance économique.

\section{A) Études révisionnistes}

Les chercheurs se sont d'abord intéressés à l'existence dans les communautés catholiques d'avant le XVII ${ }^{e}$ siècle d'un système capitaliste semblable à celui décrit par la théorie de Weber. La question est importante car elle porte sur la relation complexe entre le calvinisme austère et l'esprit capitaliste lequel a mené, d'après Weber, à l'émergence du capitalisme de production moderne dans les communautés protestantes. À cet égard, la théorie économique de l'intérêt connue sous le nom de "doctrine de l'usure", ainsi que la théorie du "prix juste» développées toutes deux par les scolastiques, sont considérées comme une indication du développement d'une philosophie et d'une économie capitaliste au moyen âge dans les communautés catholiques ${ }^{16}$. 
Le traitement de ces deux théories doit beaucoup à Saint Thomas d'Aquin qui a vécu entre 1225 et $1274^{17}$. En focalisant son attention sur les prêts monétaires, Saint Thomas s'est intéressé aux deux premières fonctions de la monnaie, à savoir, comme étalon de mesure et comme instrument d'échange. En tant qu'étalon de mesure, il considère que la valeur de la monnaie est la même que sa valeur nominale légale qui demeure constante dans le temps. Ainsi, la valeur de la monnaie est indépendante de la valeur des biens contre lesquels on peut l'échanger. Les prix de ces biens peuvent varier avec l'offre et la demande, ce qui fait fluctuer le pouvoir d'achat de la monnaie mais non sa valeur légale comme étalon de mesure, laquelle est toujours invariable. Il s'en suit que la monnaie n'est pas un bien qu'on peut acheter et vendre puisque sa valeur constante comme étalon de mesure ne doit pas être soumise aux aléas de l'offre et de la demande. Cette idée que la monnaie est non négociable est le fondement de la doctrine de l'usure.

Par ailleurs, Saint Thomas définit aussi la monnaie comme un bien fongible, c'est-à-dire un bien consomptible, comme le vin par exemple, qu'on ne peut utiliser sans le consommer. Ainsi, les services qu'on peut obtenir d'un tel bien exigent son aliénation, ce qui implique que l'utilisateur doit en être propriétaire. En s'appuyant sur cette double définition de la monnaie, à savoir comme bien fongible et comme bien non négociable, Saint Thomas argumente que tout prêt monétaire entraine nécessairement le transfert de la propriété du montant prêté lequel doit être remboursé par un montant équivalent sans plus. En exigeant un remboursement plus élevé que le montant prêté, on se trouve à réclamer deux paiements: l'un pour rembourser le montant prêté, ce qui est acceptable, et l'autre pour son utilisation par l'emprunteur, ce qui est injustifiable puisqu'on paierait pour un bien inexistant.

Mais c'est en prolongeant ses idées aux nombreux cas de contrats de société de personnes qui étaient fréquents en ce temps que Saint Thomas a présenté un nouveau principe particulièrement important pour notre discussion. Le cas qui retient notre attention est celui d'une société de deux personnes où la contribution de la première se limite à son travail et celle de la seconde se limite au financement monétaire qui pourrait prendre la forme d'un prêt. Or, il était tout à fait légitime en ce temps que les partenaires se partagent les gains et les pertes de la société. Cette situation était problématique pour les scolastiques car elle justifiait le revenu du partenaire investisseur alors qu'elle condamnait l'intérêt que recevait le créancier. 
Pour régler ce dilemme, Saint Thomas abandonne le principe de la fongibilité de la monnaie et lui substitue un nouveau principe à savoir que la propriété de l'argent investie dans l'entreprise revient au partenaire investisseur qui est seul à encourir le risque de la perdre. Autrement dit, le partenaire travailleur peut utiliser les fonds fournis par l'investisseur mais ne participe pas dans leur propriété puisqu'il n'en est pas redevable en cas de faillite. Ainsi, le partenaire investisseur conserve seul la propriété des fonds investis et participe par le fait même dans les profits de la société à titre de co- propriétaire. Cette analyse a longtemps dominé la pensée des scholastiques qui ont suivi Saint Thomas dans leur quête de justifier les profits que se partagent les partenaires d'une société, tout en condamnant le paiement d'intérêt sur les prêts.

Poussant plus loin sa réflexion, Saint Thomas se pencha sur la relation entre la valeur des commodités et leur utilité pour les personnes qui les acquièrent. Il présenta alors le principe que la valeur d'une commodité est fonction de son utilité pour l'acquéreur laquelle dépend de l'usage qu'il en fera. Autrement dit, c'est le besoin individuel qui détermine la valeur des commodités. Et même si d'autres docteurs scolastiques ont considéré les propriétés matérielles d'un bien comme une seconde composante de sa valeur, il était clair que cette dernière n'a d'effet que si ces propriétés satisfont un besoin humain en premier. En somme, la valeur d'un bien est ancrée dans son utilité pour les consommateurs individuels.

Cependant, le concept que la valeur de la commodité dépend de l'évaluation subjective de chaque individu, pose problème si on veut dériver le prix d'équilibre du marché pour cette commodité. Pour résoudre ce problème, les scolastiques ont avancé le principe que le prix d'équilibre est déterminé subjectivement par le consensus de tous les participants au marché et devient ainsi un prix objectif pour chaque participant individuel. Autrement dit, la valeur d'une commodité est déterminée en fonction des besoins de l'ensemble des acheteurs et des vendeurs de la communauté et non en fonction du besoin d'un individu en particulier. Ainsi, pour les scolastiques, la valeur est un phénomène social. En rigueur de terme, on dira que dans un marché où la concurrence est parfaite ${ }^{18}$, le prix d'équilibre d'un bien reflète sa demande et son offre globales et c'est à ce prix que les transactions individuelles se font. Il s'agit ici de la définition du "prix juste».

Il s'en suit que le "prix juste" ainsi défini comme prix d'équilibre n'est ni précis, ni fixe en pratique. Ce qui fait dire à Saint-Thomas qu'une petite différence avec le "prix juste" théorique n'est pas 
condamnable, en autant que les deux parties impliquées dans la transaction acceptent librement un prix différent du "prix juste». De plus, le "prix juste" peut varier dans le temps et dans l'espace. Par exemple, le prix d'une denrée agricole peut être élevé en début de saison et baisser par la suite avec l'augmentation de l'offre. Saint-Thomas considère alors le prix élevé et le prix inférieur subséquent comme étant tous deux des "prix justes" qui expriment la valeur à des temps différents. Dans la même veine, Saint-Thomas déclare que le prix peut augmenter si la transaction occasionne un risque additionnel pour le vendeur et aussi pour couvrir les frais de transport du bien. Il est intéressant de noter à cet égard que dans la pensée économique des scolastiques, le travail fourni par la main-d'œuvre ne rentre pas en ligne de compte dans la détermination du "prix juste».

Les doctrines de "l'usure» et du "prix juste» ne tardèrent pas à être reprises avec clarté et rigueur par deux docteurs scolastiques qui ont suivi les traces de Saint-Thomas. Il s'agit de San Bernardino de Siena (1380-1444) auteur d'un important traité sur l'économique et de Sant'Antonino de Florence (1389-1459) auteur d'une vaste étude sur la théologie morale qui lui sert aussi à mettre en évidence et à analyser des thèmes économiques ${ }^{19}$. Ces textes revêtent en effet une importance notable puisqu'ils confirment l'avancée de la pensée économique chez les scolastiques.

Selon plusieurs auteurs, ces développements théoriques font miroiter l'existence d'un système capitaliste dans les sociétés européennes catholiques du moyen âge dont les contours ont caractérisé jusqu'à récemment notre vie économique. Sur le plan théorique, Kauder déplore le fait que la théorie de l'utilité que les scolastiques et par la suite les penseurs catholiques, italiens et français, ont développée, n'ait pas été adoptée et enrichie par les penseurs protestants. Il s'agit ici d'un antagonisme apparent entre les deux écoles de pensée ${ }^{20}$. Ainsi, la tradition aristo-thomiste fait intervenir le besoin des consommateurs et la rareté des biens dans la détermination de la valeur des biens, alors que les penseurs protestants ont plutôt lié la valeur des biens à la quantité du travail fourni dans leur production, ce qui a ouvert la voie au Marxisme. De Roover abonde dans le même sens et maintient que les doctrines de «l'usure» et du "prix juste» n'étaient que le début d'un développement théorique qui a été étendu par les scolastiques à une foule d'autres questions telles que le salaire juste, l'inflation, la justice dans la taxation, la dette publique, le monopole, le taux de change, les partenariats et de façon générale tous les contrats qui peuvent avoir de près ou de loin un lien avec l'usure. De plus, il importe de souligner 
l'adoption par les scolastiques du XVIe siècle de la théorie de la quantité de la monnaie et leur préoccupation vis-à-vis la légalité des transactions bancaires. Par ailleurs, De Roover ${ }^{21}$ déplore le fait que certains auteurs dont Max Weber et Richard H. Tawney aient été influencés à tort par les travaux de Henrich von Langerstein du XIVe siècle, ce qui les a amenés à adopter une mauvaise interprétation du concept du "prix juste» et de ses extensions par les scolastiques. À cet égard, De Roover trouve aussi que les écrits des scolastiques démontrent clairement que l'Église n'a ni favorisé ni empêché le développement du capitalisme, ce qui lui fait douter de la théorie de Weber.

Robertson arrive aux mêmes conclusions dans sa revue chronologique des évènements historiques de l'époque ce qui l'amène à rejeter la théorie de Weber. Il maintient que le capitalisme a pris son envol dans les cités italiennes du XIVe siècle qui étaient de confession catholique, et que c'est dans ce contexte que le libéralisme et le rationalisme ont pris racine bien avant que dans les pays protestants ${ }^{22}$. Il argumente aussi que la théorie économique libérale a été développée dans les pays catholiques, surtout en France et en Italie, sous l'influence des scolastiques. R. H. Tawney rejette lui aussi la théorie de Weber et maintient que "l'esprit du capitalisme» est aussi vieux que le monde et qu'il n'est pas le fruit du protestantisme ${ }^{23}$.

Pour sa part, Joseph Schumpeter maintient que les sociétés féodales du moyen âge contenaient en elles-mêmes les germes d'un système capitaliste. L'émergence de ce système a procédé à petits pas en introduisant graduellement des méthodes de production capitalistes. Ainsi, on assiste à partir du XIII ${ }^{\mathrm{e}}$ siècle à un déclin graduel des institutions féodales et à une croissance parallèle des entreprises capitalistes dans les communautés européennes catholiques ${ }^{24}$. Selon Schumpeter, vers la fin du $\mathrm{XV}^{\mathrm{e}}$ siècle, tous les phénomènes associés au système capitaliste contemporain, incluant les grandes entreprises, la spéculation sur les commodités et le système financier moderne étaient déjà en place, ce qui réfute la thèse du rôle fondamental du protestantisme dans l'émergence du capitalisme de production moderne.

Henryk Grossman rejette à son tour l'idée que le calvinisme était une condition préalable nécessaire pour l'émergence du capitalisme comme le prône la théorie de Weber. Il s'appuie à cet égard sur le fait que le capitalisme s'est répandu en Italie sous le catholicisme, deux siècles avant l'apparition du protestantisme. Pour Grossman, le rôle de toute religion, qu'elle soit protestante, calviniste, catholique, janséniste, moliniste ou autre est de rendre les individus soumis à l'autorité, dévoués au travail et satisfaits de leur sort dans la vie sans chercher à l'améliorer. 
On reconnait là une définition de la religion qui semble avoir des accents marxistes. Poussant plus loin cette idée, Grossman argumente que c'est cette domestication des peuples propre à toutes les religions qui constitue la face visible de "l'esprit du capitalisme».

Plusieurs autres historiens du $\mathrm{XX}^{\mathrm{e}}$ siècle se sont également penchés sur l'ordre chronologique de l'émergence du capitalisme et du calvinisme en Europe. À cet égard, E. Fischoff est d'avis que les études montrent que le capitalisme a émergé et s'est ancré dans les mours bien avant l'arrivée du calvinisme, ce qui contredit la relation de cause à effet prônée par Weber. Samuelsson abonde dans le même sens. Il critique par ailleurs l'ambiguité des notions de puritanisme, de protestantisme et de calvinisme que Weber utilise de façon interchangeable dans ses écrits ainsi que celle de capitalisme et trouve qu'on ne peut estimer un coefficient de corrélation par les techniques statistiques usuelles entre des notions aussi vagues. Il rapporte également que dans certains cas comme en Hollande, l'arrivée du capitalisme a précédé celle du calvinisme, alors que dans d'autres cas comme en Suisse, on ne peut attribuer l'émergence du capitalisme à l'influence du calvinisme étant donné la très longue période qui sépare les deux évènements. Tout ceci mène au rejet de la théorie de Weber.

Au terme de cette analyse visant à faire le point sur la chronologie historique du développement du capitalisme en Europe, on ne peut s'empêcher de constater que cette rétrospective ne peut nous amener à conclure de façon claire à l'existence d'une relation causale entre l'implantation du calvinisme austère et l'émergence de l'esprit capitaliste qui sous-tend le système capitaliste de production. D'une part, les études théologiques nous portent à croire que l'émergence de "l'esprit du capitalisme" nécessitait une condition préalable qui est celle de l'Éthos du calvinisme austère. D'autre part, si l'on en croit les études révisionnistes, le processus capitaliste était déjà enraciné dans les communautés catholiques bien avant le XVII ème siècle. Force est donc de conclure que cette voie de recherche est sans issue. C'est donc vers des études empiriques que les chercheurs se sont dirigés pour faire avancer l'analyse.

\section{B) Études empiriques}

Parallèlement aux études révisionnistes visant à valider ou à infirmer la thèse de Weber, certains auteurs ont cherché à aborder la question à travers les analyses économétriques. Un premier groupe s'est attardé aux comparaisons entre pays ou villes de confessions religieuses 
différentes afin de vérifier l'existence d'une corrélation entre la religion et le progrès économique, alors qu'un second groupe a focalisé sur l'étude des communautés à l'intérieur d'un même pays ou région.

\section{1 - Comparaisons interpays}

Dans ce groupe, Blum et Dudley analysent un échantillon de 316 villes européennes comprenant 90 villes protestantes du nord et 226 villes catholiques du sud pour les années 1700 à 1750. À la suite de De Long et Shleifer, ils utilisent les taux de croissance des populations de ces villes comme indicateurs des taux de progression des revenus. Sur la base de leurs résultats, ils rejettent l'hypothèse de la relation causale entre le protestantisme et la croissance économique prônée par Weber, comme ils rejettent aussi le rôle du capital humain à cet égard. Par contre, ils argumentent que la proximité des villes protestantes des ports de l'Atlantique et des centres d'affaires londoniens leur a permis de profiter des réseaux d'information qui n'étaient pas disponibles aux villes catholiques du sud, et de développer ainsi leurs économies plus rapidement. Dans ce même esprit, Iannaccone ainsi que Delacroix et Nielsen trouvent aussi que les résultats des comparaisons entre pays ne révèlent aucune relation causale entre le protestantisme et l'émergence du capitalisme de production, ce qui les amène à rejeter la théorie de Weber. Acemoglu, Johnson et Robinson arrivent à la même conclusion dans leur étude entre pays même après avoir contrôlé l'effet des institutions économiques.

Poursuivant sur la même lancée, Korotayev, Malkov et Khaltourina ont cherché à examiner l'impact de l'alphabétisation sur le progrès économique des protestants et des catholiques vers la fin du XIX ${ }^{\mathrm{e}}$ et début du $\mathrm{XX}^{\mathrm{e}}$ siècle en Europe. Les auteurs formulent l'hypothèse que l'encouragement du clergé protestant à leurs fidèles de lire la Bible a eu pour effet d'améliorer le capital humain protestant ce qui a conduit au progrès économique. Pour tester cette hypothèse, ils régressent le taux d'alphabétisation ${ }^{25}$ en 1800 sur le produit intérieur brut per capita en 2000 pour un échantillon de 15 pays incluant des pays non chrétiens et trouvent que le pourcentage d'alphabétisation explique $86 \%$ de la croissance économique observée. Les auteurs concluent que ce résultat confirme indirectement la théorie de Weber car l'alphabétisation des fidèles protestants qui a amélioré leur capital humain a été le fruit d'une motivation religieuse, soit la lecture de la Bible.

Dans leur étude, Barro et McCleary font la distinction entre les croyances religieuses (comme la croyance dans le ciel, l'enfer et la vie éternelle) et la fréquentation des lieux de culte (comme l'église) afin 
d'examiner l'impact de la pratique religieuse d'une part et les croyances religieuses d'autre part sur la croissance économique. Une hypothèse souvent invoquée à cet égard est que le développement économique fait baisser la vocation religieuse dans le sens qu'il a pour effet que les individus deviennent moins croyants et moins pratiquants. Les auteurs se penchent plutôt sur le lien causal inverse qui va de la vocation religieuse à la croissance économique. Ils considèrent alors que la fréquentation des lieux du culte d'une part et les croyances religieuses d'autre part, comme deux variables indépendantes dans leur modèle d'analyse. Ils utilisent ensuite des données recueillies par sondage entre 1981 et 1999 sur 41 pays dont quelques-uns sont non chrétiens et trouvent que la croissance économique est positivement corrélée avec les croyances religieuses mais négativement corrélées avec la fréquentation des lieux du culte ${ }^{26}$. Autrement dit, les croyances religieuses façonnent les caractéristiques personnelles des individus et agissent de la sorte sur la croissance économique, ce qui constitue une confirmation de la théorie de Weber.

Niall Ferguson procède à partir d'une comparaison circonstancielle entre l'attitude au travail et à la religion aux États-Unis et en Europe occidentale protestante pour vérifier la théorie de Weber. Il se penche alors sur l'écart de productivité entre ces deux économies qu'il attribue principalement à la différence marquée entre les heures travaillées des deux côtés de l'Atlantique. Citant des données de l'OCDE couvrant la période de 1973 à 1998, Ferguson rapporte que la semaine de travail s'est allongée et le taux de participation à l'emploi a progressé aux États-Unis durant cette période alors que dans les pays protestants de l'Europe occidentale ces deux variables ont suivi une trajectoire inverse. Ferguson maintient que ces deux attitudes opposées vis-à-vis du travail des deux côtés de l'Atlantique coïncident avec une baisse marquée de la pratique religieuse en Europe protestante ${ }^{27}$, alors que la pratique religieuse protestante est demeurée bien vivante aux États-Unis, ce qui corrobore la corrélation entre la vocation religieuse et l'activité économique prônée par la théorie de Weber. De toute évidence, cette observation anecdotique manque de rigueur scientifique et néglige tous les facteurs sociaux culturels qui peuvent expliquer ces différences.

À cet égard, Daniel S. Hamermesh a comparé la moyenne des heures travaillées annuellement par travailleur aux États-Unis avec celles en Australie, en France, au Canada, en Allemagne, au Japon, en Suède et en Grande Bretagne pour la période 1979 à 2015. Il note que bien que cette moyenne ait diminué dans tous ces pays au cours de cette période, la diminution aux États-Unis a été comparativement plus faible. Cet écart Hamermesh l'explique principalement par le nombre de jours de 
congés fériés qui est plus petit et par les vacances annuelles qui sont plus courtes aux États-Unis par rapport aux autres pays de l'échantil$\operatorname{lon}^{28}$. Pour ce qui est de l'attitude au travail, Hamermesh applique la logique économique pour analyser le partage du temps entre le travail et le loisir dans les sociétés économiquement développées. Il part du principe que le temps est une ressource rare et que le coût d'option du loisir ${ }^{29}$ est fonction de la rémunération du travail: plus cette rémunération est élevée, plus grand est le coût d'option du loisir. Il s'en suit que plus le revenu du travail est élevé, plus les individus sont motivés à y consacrer plus d'heures ${ }^{30}$ et à participer davantage au marché du travail en coupant sur le loisir, ce qui rend l'élasticité de l'offre globale du travail positive. En analysant les enquêtes publiées à partir des agendas de l'emploi du temps d'un échantillon d'individus de la France, de l'Allemagne et de la grande Bretagne ${ }^{31}$ Hamermesh arrive à des résultats qui confirment son hypothèse. Il convient de noter que les principes d'éthique religieuse ne jouent aucun rôle dans cette explication de la motivation au travail.

Comme on peut le constater, les comparaisons interpays donnent des résultats mitigés concernant le lien direct entre la religion et la croissance économique. De plus, ces comparaisons semblent favoriser l'introduction d'autres facteurs sociaux et culturels dans la relation sous étude. Cependant, comme l'ont fait remarquer certains chercheurs, les comparaisons entre pays peuvent être remises en question étant donné que l'effet de la religion sur la croissance économique ne peut être isolé des autres effets institutionnels, politiques et géographiques qui varient d'un pays à l'autre. Plus particulièrement, le ratio de dépendance $^{32}$ qui diffère entre les pays a une incidence capitale sur l'attitude au travail des individus et sur les programmes gouvernementaux qui s'adressent à la main-d'œuvre. En effet, plus la population d'un pays est vieillissante, plus sa contribution au travail diminue et plus les ressources gouvernementales sont dirigées vers les budgets de la santé et des régimes des rentes plutôt que vers la formation de la maind'œuvre. Pour toutes ces raisons, il serait souhaitable de centrer les comparaisons sur des communautés de confessions religieuses différentes, mais appartenant à un même pays, afin d'obtenir des résultats plus probants.

\section{2 - Études intrapays}

Dans une étude intéressante portant sur la Grande Bretagne, Beaudreau présente une théorie qui peut servir de complément à celle de Weber. Selon cette théorie, l'exode des Huguenots, suite à la révocation de 
l'Édit de Nantes en 1685 et surtout l'arrivée de 100000 à 140000 de ces derniers en Angleterre et en Irlande, et la création d'un réseau commercial étendu grâce à leur diaspora à travers l'Europe, tout ceci a contribué de façon importante à la propagation du Calvinisme et au processus économique et social qui a mené à l'émergence du capitalisme dont parle Weber ainsi qu'à la révolution industrielle. Pour vérifier sa théorie, Beaudreau développe un modèle à deux équations, l'une pour la demande globale et l'autre pour l'offre globale dans lesquelles il introduit une variable qui dénote l'impact de l'arrivée des réfugiés Huguenots avec leur savoir-faire distinctif en Angleterre et en Irlande. Dans ce cadre, le savoir-faire huguenot amène des innovations technologiques qui augmentent l'offre globale alors que le réseau commercial de la diaspora augmente la demande globale, ce qui résulte, selon les prédictions du modèle, en un accroissement de la production nationale qu'on mesure sur trois générations successives afin de tenir compte de l'intégration graduelle des réfugiés huguenots. Les données de la croissance du produit intérieur brut, du revenu per capita et de la taille de la population en Angleterre pour les années 1700 à 1870, rapportées par Beaudreau, confirment les prévisions de son modèle. Il convient de mentionner cependant que le rôle de la religion dans ce modèle est tout au plus implicite du fait qu'il caractérise tout simplement les Huguenots.

Prenant aussi le contrepied des études interpays, Becker et WöBmann limitent leur étude empirique à la Prusse du XIX ${ }^{\mathrm{e}}$ siècle. Ce choix s'est imposé surtout parce que la Prusse représente le lieu de naissance de Weber en plus d'être la région dans laquelle la réforme a germé. Par ailleurs, la Prusse avait des lois et des institutions uniformes et sa population était composée de deux tiers de protestants et un tiers de catholiques, ce qui exclut la présence de petits groupes minoritaires. L'originalité de la recherche de Becker et WöBmann repose sur le fait qu'elle porte sur une analyse empirique entre le protestantisme, l'alphabétisation et le progrès économique. Ils utilisent les données du recensement de la population de 1871 pour obtenir des informations sur l'affiliation religieuse et l'alphabétisation ainsi que des données sur le recensement des travailleurs dans les divers secteurs de l'économie de 1882 pour obtenir un indicateur du développement économique. Les résultats de leur étude montrent tout d'abord, contrairement aux comparaisons entre pays, qu'il existe une forte corrélation entre le protestantisme et la prospérité économique, ce qui confirme ce premier aspect de la théorie de Weber. Leurs résultats montrent aussi une forte corrélation entre le protestantisme et l'alphabétisation. Et lorsque le 
protestantisme et l'alphabétisation sont utilisés simultanément comme variables explicatives, seule l'alphabétisation ressort comme explication significative de la prospérité économique.

Davide Cantoni focalise sa recherche sur un milieu allemand bien délimité de 272 villes culturellement homogènes, soit les terres allemandes du Saint Empire Romain. À cet égard, sa recherche s'inscrit dans le cadre de celle de Becker et WöBmann, mais y diffère en étant une analyse de séries chronologiques couvrant les années 1300 à 1900 au lieu d'être une analyse en coupe instantanée couvrant l'année 1871. Cantoni utilise comme variable dépendante, la taille des 272 villes sous étude qu'il considère comme indicateur de leur développement économique ${ }^{33}$. Les résultats de son étude ne montrent de façon systématique aucune différence dans la performance économique à long terme entre les régions catholiques et protestantes sous étude, ce qui l'amène à rejeter l'hypothèse de l'influence du protestantisme sur la croissance économique.

Par ailleurs, un nouveau développement théorique prôné par un grand nombre de chercheurs, dont par exemple Spolaore, Mokyr, Huntington ou Inglehart et Baker, maintient que l'analyse de l'émergence du capitalisme doit prendre en compte l'évolution culturelle ancrée dans l'histoire des communautés étudiées, plutôt que limiter l'analyse aux explications traditionnelles de croissance exogène et endogène. Ces études récentes cherchent à expliquer les facteurs déterminants de la croissance économique à partir de variables culturelles fondamentales dont les racines remontent très loin dans l'histoire des peuples. Rappelons à ce stade-ci que le concept de culture se prête mal à une définition précise. Ainsi on retrouve, dans la littérature, différentes définitions proposées par divers auteurs. Pour les besoins de notre étude, nous définirons la culture, à l'instar de Spolaore ${ }^{34}$, comme un ensemble d'attributs - tels que les valeurs, les traditions et les croyances - qui ne sont pas transmises génétiquement d'une génération à l'autre d'une même société, mais qui sont plutôt acquises par l'apprentissage et l'imitation. À cet égard, McClelland trouve que les communautés dans lesquelles la réussite économique constitue un accomplissement positif ancré dans les mœurs et transmis de génération en génération, réalisent des taux plus élevés de croissance de leur économie que les communautés qui n'ont pas cette culture. Landes aussi suggère que l'explication de la divergence entre les pays du nord et du sud de l'Europe avant la révolution industrielle est probablement causée par une divergence de l'évolution culturelle. D'après lui, les facteurs culturels représentent la clé pour expliquer les divergences du taux de croissance économique des divers pays. 
Selon Mokyr, l'origine intellectuelle de la révolution industrielle remonte à Francis Bacon et ses disciples qui ont préconisé au $\mathrm{XVII}^{\mathrm{e}}$ siècle un programme de recherche empirique lequel fut mis en place au XVIII ${ }^{\mathrm{e}}$ siècle en Europe occidentale, en s'appuyant sur un développement culturel et institutionnel, ce qui a préparé le terrain pour la croissance économique qui a suivi. Spolaore et Wacziarg abondent dans le même sens en identifiant un choc culturel spécifique - notamment l'émergence d'une approche scientifique de la recherche qui se fonde sur la preuve empirique - laquelle ils considèrent la cause fondamentale du changement de culture qui a mené à l'industrialisation. Ils concluent que toute explication de la révolution industrielle qui exclut l'évolution culturelle est incomplète.

Dans ce même esprit, Ganato, Inglehart et Leblang construisent un indice culturel, appelé "indice de motivation de la réussite économique", qui est composé de quatre variables dont deux sont positives et les deux autres sont négatives. Cet indice est défini comme suit:

(Frugalité + détermination) - (obéissance + conviction religieuse).

Ils vérifient la relation entre cet indice et le taux de croissance du PIB de 25 pays de 1960 à 1989, et trouvent que l'indice explique plusieurs aspects de la croissance économique qui ne sont pas pris en compte par les variables économiques des modèles de croissance endogène et exogène traditionnels. D'autre part, à partir d'une enquête menée aux États Unis, Alwin rapporte une énorme différence entre les communautés protestantes et catholiques, en ce qui concerne les valeurs enseignées aux enfants. Il trouve cependant que ces différences s'estompent avec le temps et que les enseignements dans les deux communautés finissent par converger vers un système de valeurs communes.

Cette discussion des études intrapays nous montre que la prise en compte de la réalité sociale et culturelle des diverses communautés d'un même pays, plus spécialement leurs valeurs morales, leur appui à l'éducation et leur ouverture sur le monde, permet une meilleure compréhension du problème de la croissance économique en mettant en évidence les multiples facteurs qui peuvent contribuer au développement d'un esprit capitaliste. Plus important encore, les résultats de ces études laissent entrevoir que l'influence de ces facteurs sur l'émergence d'un esprit capitaliste n'est pas unidimensionnelle mais s'opère plutôt avec rétroaction. Il s'agit là d'un élément important à introduire dans les modèles économétriques. 


\section{CONCLUSION}

Weber a introduit l'idée d'une relation entre les doctrines de la destinée et de la vocation au travail véhiculées par le calvinisme austère du XVII ${ }^{\mathrm{e}}$ siècle d'une part et "l'esprit du capitalisme" qui a émergé dans les communautés protestantes de l'époque d'autre part et qui a favorisé leur adhésion au capitalisme et conséquemment leur supériorité économique par rapport aux communautés catholiques. Depuis son travail de pionnier, un grand nombre de chercheurs provenant de disciplines différentes ont repris cette théorie l'interprétant comme établissant un lien causal entre l'éthique calviniste et l'esprit capitaliste, et l'ont analysée tantôt pour démontrer sa validité, tantôt pour la rejeter. À cette fin, deux approches de recherche différentes ont été adoptées, soit l'approche révisionniste d'une part et celle de l'analyse statistique des comparaisons entre pays ou entre les diverses communautés d'un même pays d'autre part.

Le tour d'horizon des principales études des six dernières décennies sur la théorie de Weber renvoie un constat clair pour les recherches futures. Quel que soit l'approche scientifique utilisée, il est important de ne pas aborder la question d'une façon réductrice en se limitant uniquement à un seul facteur causal, soit le facteur religieux et en se limitant à une relation unidimensionnelle et sans rétroaction. En effet, tel que mentionné dans l'introduction, Weber a reconnu dans sa thèse la complexité de cette relation et a préconisé, à certains égards, une conjonction de fait plutôt qu'un lien causal direct entre l'éthique calviniste et «l'esprit du capitalisme». Ainsi, pour appréhender le réel dans sa complexité, sa pluralité et ses incontournables nuances, il est primordial de l'analyser sous ses multiples angles, parfois enchevêtrés, dont les interférences varient selon les situations. Il s'agit alors d'expliquer l'inégalité des revenus et des richesses qui a pris son envol au XVII ${ }^{e}$ siècle entre les communautés protestantes et catholiques en tenant compte dans l'analyse de l'évolution de plusieurs facteurs culturels dont principalement les facteurs démographiques, sociaux, politiques, scientifiques, éthiques et religieux - qui s'influencent mutuellement. En élargissant ainsi le champ d'analyse au-delà des modèles exogènes et endogènes de croissance économique, on peut évaluer le potentiel de chaque communauté à partir duquel elle peut développer son économie. C'est dans cette direction que la recherche scientifique récente s'est dirigée, ce qui pourrait aboutir à une théorie générale qui prendrait en compte simultanément tous les facteurs qui ont contribué à l'implantation du processus capitaliste, et qui mettrait ainsi en lumière les facteurs qui conditionnent le potentiel de la croissance économique. 


\section{RÉFÉRENCES}

[1] Acemoglu, Daron, Simon Johnson \& James A. Robinson, The Colonial Origins of Comparative Development: An Empirical Investigation, American Economic Review, 2001, Vol. 91 (5), p. 1369-1401.

[2] Allen, Robert C., The Great Divergence in European Wages and Prices from the Middle Ages to the First World War, Explorations in Economic History, 2001, Vol. 38, p. 411-447.

[3] Alwin, Duane F., Religion and Parental Child-Rearing Orientations: Evidence of a Catholic-Protestant Convergence, American Journal of Sociology, 1986, Vol. 92, p. 412-440.

[4] Bairoch, Paul, Taille des villes, conditions de vie et développement économique, Paris: Éditions de l'École des Hautes Études en Sciences Sociales, 1977.

[5] Barro, Robert J. \& Rachel M. McCleary, Religion and Economic Growth across Countries, American Sociological Review, October 2003, p. 760-781.

[6] Barro, Robert J., Economic growth in a Cross Section of countries, Quarterly Journal of Economics, 1991, Vol. 106, p. 407-444.

[7] Beaudreau, Bernard C., Calvinism, Huguenots and the Industrial Revolution, Department of Economics, Université Laval, 2019, 46 pages.

[8] Becker, Sascha O. \& Ludger WöBmann, Was Weber Wrong? A Human Capital Theory of Protestant Economic History, Quarterly Journal of Economics, May 2009, Vol. 12 (2), p. 531-596.

[9] Blum, U. et Leonard Dudley, Religion and Economic Growth: Was Weber Right?, Journal of Evolutionary Economics, 2001, Vol. 11, p. 207-230.

[10] Boettcher, Susan R., Confessionalization: Reformation, Religion, Absolutism, and Modernity, History Compass, January 2004, Vol. 2 (1), p. $1-10$.

[11] Braudel, Fernand, Civilization and Capitalism 15 th $-18^{\text {th }}$ Century, vol. 1, The Structures of Everyday Life, New York: Harper and Row, 1981.

[12] Cantoni, Davide, The Economic Effets of the Protestant Reformation: Testing the Weber Hypothesis in the German Lands, Job market paper, Harvard University, November 2009.

[13] Chodorov, F., The Radical Rich, Article excerpted from chapitre 19 of "Out of Step" 1962, http://media.mises.org/mp3/audioarticles/5031_ chodorov.mp3 
[14] Delacroix, Jacques et F. Neilsen, The Beloved Myth: Protestantism and the Rise of Industrial Capitalism in Nineteenth-Century Europe, Social Forces, December 2001, Vol. 80 (2), p.509-553.

[15] De Long, J. Bradford \& Andrei Shleifer, Princes and Merchants: European City Growth before the Industrial Revolution, Journal of Law and Economics, 1993, Vol. 36, p. 671-701.

[16] De Roover Raymond, Scholastic Economics: Survival and Lasting Influence from the $16^{\text {th }}$ century to Adam Smith, Quarterly Journal of Economics, May 1955, p. 161-190.

[17] De Roover Raymond, The Concept of the Just Price: Theory and Economic Policy, The Journal of Economic History, Vol. 18 (4), December 1958, p. $418-434$.

[18] De Roover Raymond, San Bernardino of Siena and Sant'Antonino of Florence: The Two Great Economic Thinkers of the Middle Ages, Baker Library, Harvard Graduate School of Business Administration, 1967, 46 pages.

[19] Dudley, Leonard Communications and Economic Growth, European Economic Review, 1999, Vol. 43 (3), p. 595-619.

[20] Fergusson, Niall, The World; Why America Outpaces Europe (Clue: The God Factor), the New York Times, June 8, 2003.

[21] Fischoff E., The Protestant Ethic and the Spirit of Capitalism: the History of a Controversy, 1944.

[22] Graff, Michael, Educational Imbalance, Socio-Economic Inequality, Political Freedom, and Economic Development, Journal of Economic Development, Vol. 24 (2), 1999.

[23] Granato, Jim, R. Inglehart et Leblang D., The effect of Cultural Values on Economic Development: Theory, Hypotheses and some Empirical Tests, American Journal of Political Science, August 1996, Vol. 40 (3), p. 607-631.

[24] Grier, Robin, The effect of Religion on Economic Development: A Cross National Study of 63 Former Colonies, Kyklos, February 1997, Vol. 50 (1), p. $47-62$.

[25] Guiso, Luigi, Paola Sapienza et Luigi Zingales, People's Opium? Religion and Economic Attitudes, Journal of Monetary Economics, 2003, Vol. 50, p. 225-282.

[26] Guiso Luigi, Paola Sapienza et Luigi Zingales, Does Culture Affect Economic Outcomes?, Journal of Economic Perspectives, Spring 2006, Vol. 20 (2), p. 23-48. 
[27] Grossman, Henryk, The Beginnings of Capitalism and the New Mass Morality, Journal of Classical Sociology, 6(2), July 2006.

[28] Hamermesh S. Daniel, Spending Time, The Most Valuable Resource, Oxford University Press, 2019.

[29] Hénaff Marcel, Religious Ethics, Gift Exchange and Capitalism, Arch. Europ. Sociol., XLIV, 2003, p. 293-324.

[30] Hénaff Marcel, The Price of Truth, Stanford University Press, Stanford, California, 2010, p. 269-276.

[31] Huntington, S.P., The Clash of Civilizations and the Remaking of World Order, New York, Simon \& Schuster, 1996.

[32] Iannaccone, L.R., The Consequences of Religious Market Structures: Adam Smith and the Economics of Religion, Rationality and Society, April 1991, p. 156-177

[33] Inglehart, R. \& W.E. Baker, Modernization, Cultural Change and the Persistence of Traditonal Values, American Sociological Review, February 2000, p. 19-51.

[34] Kauder Emil, The retarded Acceptance of the Marginal Utility Theory, Quarterly Journal of Economics, November 1953, p.564-575.

[35] Khoury, Nabil, La condamnation de l'usure dans le Christianisme et dans l'Islam, Assurances et Gestion des risques, juin 2018, Vol. 85 (1-2), p. 1-25.

[36] Korotayev, A., A. Malkov \& D. Khaltourina, Introduction to Social Macrodynamics, Moscow: URSS, ISBN 5-484-00414-4, Chapitre 6, Reconsidering Weber: Literacy and the Spirit of Capitalisme, 2006, p. 87-91.

[37] Landes, David, 2000, Culture Makes Almost All the Difference, dans Culture and Economic Growth, Enrico Spolaore (ed.), An Algar Research Collection, 2014, Vol. I p. 105-117.

[38] Mankiw, N. Gregory, David Romer et David N. Weil, A contribution to the Empirics of Economic Growth, Quarterly Journal of Economics, 1992, Vol. 107, p. 407-437.

[39] McClelland, David, The achieving Society, Free press, paperback edition 1967.

[40] Merton, Robert K., Science, Technology and Society in Seventeenth Century England, Osiris, University of Chicago Press, 1938, Vol. 4, p. 360-632. 
[41] Mokyr, Joel, 2005, The Intellectual Origins of Modern Economic Growth, dans Culture and Economic Growth, Enrico Spolaore (ed.), An Algar Research Collection, 2014, Vol. I p. 758-824.

[42] Robertson, Hector M., A Criticism of Max Weber and his School, Second Edition, Edited by Robert W. Green, D.C. Heath and Company, 1973, p. 53-88.

[43] Samuelsson, Kurt, Religion and Economic Action, New York: Basic Books, 1961.

[44] Samuelsson, Kurt, A Criticism of Max Weber and his School, in "Protestantism, Capitalism, and Social Science», Second Edition, Edited by Robert W. Green, D.C. Heath and Company, 1973, p. 53-88.

[45] Schumpeter, J.A., History of Economic Analysis, Taylor and Francis, e-library, 2006.

[46] Spolaore Enrico \& Romain Wacziarg, 2013, How Deep Are the Roots of Economic Development?, dans Culture and Economic Growth, Enrico Spolaore (ed.), An Algar Research Collection, 2014, Vol. II p. 3-47.

[47] Tawney R. H., Religion and the Rise of Capitalisme, in Protestantism, Capitalism and Social Science, Second Edition, Edited by Robert W. Green, D.C. Heath and Company, 1973, p. 89-105.

[48] Taylor, Charles, Les sources du moi: la formation de l'identité modern, les éditions du Boréal, 1998, chapitres 13, 14 et 17.

[49] Viner, Jacob, Religious Thought and Economic Society, Duke University Press, 1978, chapitre 4.

[50] Weber, Max, L'éthique protestante et l'esprit du capitalisme, Collection dirigée par Oliver Amiel, Études de sociologie de la religion, Édition Plon 1964.

\section{NOTES}

1 Professeur Émérite, Université Laval, ntkhoury@hotmail.ca. Je suis redevable aux Professeurs Bernard C. Beaudreau, Patrice Bergeron, Jean-Marie Gagnon, Michel Roland, Philippe Roy-Lysencourt et un arbitre anonyme pour leurs commentaires qui ont beaucoup amélioré la qualité du texte.

2 La «International Sociological Association» a classé cet ouvrage comme le quatrième plus important livre publié au XXe siècle. Voir : Elliot Green,12 May, 2016, What are the Most-cited Publications in the Social Sciences (according to Google scholar)? LSE Imact Blog, London School of Economics.

3 Voir Max Weber, L'Éthique protestante et l'esprit du capitalisme, chapitre 1, p. 43-80 et chapitre 2, p. 219-227. 
4 Robertson avance l'idée que Weber a voulu, par sa théorie, inverser le rapport de cause à effet que Marx avait établi pour l'interprétation historique de l'évolution du système économique. Voir H.M. Robertson, A criticism of Max Weber and his School, p. 53-88.

5 Marcel Hénaff affirme pour sa part que la relation préconisée par Weber n'a jamais été entre la foi protestante et le progrès économique, mais plutôt entre l'éthique protestante et «l'esprit du capitalisme », et que Weber n'a jamais avancé l'idée que cette relation était causale dans un sens ou dans l'autre mais qu'elle était plutôt conjoncturelle. Voir : Marcel Hénaff, The Price of Truth, p. 269-276.

6 II convient de préciser que même si Calvin s'est beaucoup intéressé à la doctrine de la prédestination, celle-ci a été principalement diffusée par les générations qui lui ont succédé. En effet, la doctrine a également connu des débats vigoureux après Calvin et surtout au Synode de Dordrecht (1618-1619), de la confession de foi de Westminster (1646) et de la Déclaration de Savoie (1658). Voir : Pierre Chanu : Le temps des réformes, Paris, Fayard, 1975. Je suis redevable au Professeur Patrice Bergeron pour cette précision.

7 Voir: C. Taylor, Les sources du moi, chapitres 13, 14 et 17.

8 II convient de souligner que Weber ne référait pas dans sa théorie à l'authentique doctrine de Calvin, mais plutôt à la version de cette doctrine qui prévalait au 17e siècle et qu'il appelait parfois le «calvinisme corrompu ». Voir Jacob Viner, Religious Thought and Economic Society, p. 154.

9 L'argument de Weber s'appuie sur l'approche de la "conjoncture prétérite» qui, dans le cas présent, consiste à présumer le comportement probable d'un individu qui agirait selon ses croyances. II s'agit bien entendu d'un argument subjectif. Voir : Viner, op.cit. p. 156.

10 Weber maintient que sa thèse s'applique uniquement aux communautés qui adoptent le protestantisme austère. II inclut dans ce groupe les calvinistes, les réformés, les baptistes, les zwinglians, les mennonites, les quakers, les méthodistes et les luthériens piétistes. Ce groupe exclut notamment les anglicans, la majorité des luthériens et bien entendu les catholiques romains. Voir : Max Weber : L'éthique protestante et l'esprit du capitalisme, p.109-185 et 231-263.

11 Hénaff souligne que dans son livre Weber ne donne pas une définition du capitalisme, mais donne seulement une définition de «l'esprit du capitalisme» qu'il désigne non pas comme l'envie d'acquérir mais plutôt la maîtrise rationnelle de ce reflexe primaire. Ainsi, l'essentiel de «l'esprit capitaliste » serait de se consacrer à la recherche d'investissements lucratifs et d'occasions d'affaires profitables en développant les échanges commerciaux, en s'employant à utiliser les connaissances et les techniques nouvelles pour augmenter la productivité de la main-d'œuvre, en s'impliquant dans le renforcement du droit à la propriété, en un mot en cherchant à consolider l'activité économique et sociale à tous les niveaux. Voir : Hénaff, op. cit. p. 298.

12 Voir R. K. Merton: Science, Technology and Society in Seventeenth Century England, p.444.

13 Marcel Hénaff fait remarquer que Weber mentionne la situation des populations catholiques sans l'expliquer. II attribue tout simplement leur comportement au refus du protestantisme austère. Voir : Hénaff, op. cit. p. 294.

14 Voir : Viner, op. cit. p. 156

15 Voir: Viner, op. cit. p. 152.

16 L'analyse de la doctrine de l'usure et de la théorie du prix juste s'inspirent beaucoup de l'article de Nabil Khoury, La condamnation de l'usure dans le Christianisme et dans I'Islam, p. 7-9.

17 Voir à titre d'exemple: Raymond De Roover, Scholastic Economics: Survival and Lasting Influence from the 16th Century to Adam Smith, p. 162.

18 Un marché dans lequel aucun acheteur ou vendeur n'a d'influence sur la formation du prix, les transactions se font sans frais ni taxes, l'information complète est disponible à tous les participants gratuitement et elle est complètement et instantanément reflétée dans les prix.

19 Voir : R. De Roover, San Bernardino of Siena and Sant'Antonino of Florence, The Two Great Economic Thinkers of the Middle Ages, p. 16-33.

20 D'après Kauder, l'école de pensée catholique incluait les penseurs suivants: Lottini, Davanzatti, Montanari, Galian, Beccaria, Turgot et Condillar, alors que l'école de pensée protestante incluait: Petty, Locke et Adam Smith. Voir : Emil Kauder, The Retarded Acceptance of the Marginal Utility Theory, p. 565. 
21 Voir: R. De Roover, The concept of the Just Price: Theory and Economic Policy, p. 418-426. Je suis redevable à l'arbitre anonyme pour cette précision.

22 Plus particulièrement, Robertson affirme que la contribution des calvinistes à «l'esprit du capitalisme » était la même que celle des jansénistes qui encourageaient le travail, la frugalité, I'honnêteté et la discipline, alors que les Jésuites sont même allés plus loin et ont favorisé l'entrepreneurship, la spéculation et l'accroissement du commerce pour le bien de la société. Robertson va même jusqu'à dire que la confession religieuse qui a déclenché l'esprit du capitalisme n'était pas le calvinisme, mais plutôt le Jésuitisme. Voir : H.M. Robertson, Aspects of the Rise of Economic Individualism, a Criticism of Max Weber and his School, p. 164.

23 Voir: R.H. Tawney, Religion and the Rise of Capitalism, p. 89-105.

24 Voir: Joseph Schumpter, History of Economic Analysis, p. 74-111.

25 C'est-à-dire le pourcentage de la population adulte qui est alphabète.

26 À cet égard, les auteurs font remarquer que la fréquentation des lieux de culte absorbe des ressources productives. Son impact sur la croissance économique dépend alors du niveau des croyances économiques qu'elle peut générer.

27 Ferguson rapporte que moins de $10 \%$ des fidèles en Hollande, Angleterre, Allemagne, Suède et Danemark fréquentent l'église une fois par mois. Voir : Niall Ferguson, The World; Why America Outpaces Europe (Clue: The God Factor), p.3.

28 Voir : Daniel S. Hamermesh, Spending Time: The Most Valuable Ressource, p. 37-41.

29 Le coût d'option se définit comme le profit qu'on aurait pu obtenir de la plus rentable activité concurrentielle qu'on doit sacrifier en faveur de celle qu'on a choisie.

30 Hamermesh cite, à titre d'exemple, trois professions qui sont mieux rémunérées que les autres en vertu de la formation poussée qu'elles nécessitent, à savoir : les professeurs d'université qui travaillent 6 heures par semaine de plus que la moyenne américaine, les médecins qui en travaillent 10 heures de plus et les avocats qui y mettent 3 heures de plus. Voir : Hamermesh, op. cit. p. 145-146.

31 En France, il s'agit de Enquête emploi du temps 2009-2010, environ 24000 agendas; en Allemagne, il s'agit de Zeitverwendungserhebung, 2012-2013, environ 25000 agendas; et en Grande Bretagne, il s'agit de Time Use Survey, 2014-2015, environ 16000 agendas. Voir : Hamermesh, op.cit. p. IX.

32 De façon générale, il s'agit du nombre total d'individus âgés de plus de 65 ans ainsi que de ceux ayant moins de 16 ans par rapport au nombre total de ceux âgés entre 16 et 65 ans.

33 Cantoni s'appuie sur le recensement manufacturier prusse des années 1816 à 1821 pour justifier son hypothèse d'une relation entre la taille des villes et le développement économique.

34 Voir E. Spolaore: Culture and Economic Growth, Volume I, p. XIII. 